\title{
Pre-dispensed Inhalation Powder Dosage
} Form

National Cancer Institute

\section{Source}

National Cancer Institute. Pre-dispensed Inhalation Powder Dosage Form. NCI

Thesaurus. Code C149580.

Solid preparation intended for inhalation use, consisting of one or more powders of solid active substance(s) presented in a suitable pharmaceutical form other than a hard capsule, either in the form of a single dose or divided into multiple single doses. The preparation is loaded into a dry-powder inhaler to generate an aerosol. 